\title{
Eradication of Ebola Based on Dynamic Programming
}

\author{
Jia-Ming Zhu, ${ }^{1}$ Lu Wang, ${ }^{2}$ and Jia-Bao Liu ${ }^{3}$ \\ ${ }^{1}$ School of Statistics and Applied Mathematics, Anhui University of Finance and Economics, Bengbu 233030, China \\ ${ }^{2}$ School of Finance, Anhui University of Finance and Economics, Bengbu 233030, China \\ ${ }^{3}$ School of Mathematics and Physics, Anhui Jianzhu University, Hefei, Anhui 230601, China
}

Correspondence should be addressed to Jia-Bao Liu; liujiabaoad@163.com

Received 5 March 2016; Revised 11 April 2016; Accepted 17 April 2016

Academic Editor: Chung-Min Liao

Copyright (C) 2016 Jia-Ming Zhu et al. This is an open access article distributed under the Creative Commons Attribution License, which permits unrestricted use, distribution, and reproduction in any medium, provided the original work is properly cited.

\begin{abstract}
This paper mainly studies the eradication of the Ebola virus, proposing a scientific system, including three modules for the eradication of Ebola virus. Firstly, we build a basic model combined with nonlinear incidence rate and maximum treatment capacity. Secondly, we use the dynamic programming method and the Dijkstra Algorithm to set up M-S (storage) and several delivery locations in West Africa. Finally, we apply the previous results to calculate the total cost, production cost, storage cost, and shortage cost.
\end{abstract}

\section{Introduction}

The Ebola virus large outbreaks in Africa began in 2014. The high number of people infected and the high mortality caused widespread concern in the world. Ebola virus disease shocked the world in 1976. It turned up for the first time in two cases that began the epidemic at the same time [1]. Another one is in the Democratic Republic of the Congo, which occurred near the Ebola River in a village [2].

Now the spread of Ebola virus has caused wide public concern all over the world. In order to make drugs and vaccine exert the greatest effect which can effectively cure patients, we propose a scientific system, including three modules for the eradication of Ebola virus. First, we build a basic model combined with nonlinear incidence rate and maximum treatment capacity. Then, we use the dynamic programming method and the Dijkstra Algorithm to set up M-S (storage) and several delivery locations in West Africa. Finally, we apply the previous results to calculate the total cost, production cost, storage cost, and shortage cost.

We established a practical, sensitive, useful model. For other manuscripts, we find that iteration, Floyd algorithm, and genetic algorithm are used to optimize the eradication of Ebola. Meanwhile, in our model, we consider not only the disease propagation speed and the drugs required quantity and the impact of transportation on the treatment but also the design of a distribution optimization feasible transmission system [3]. In addition, transmission sites and vaccine and drug production speed are points we should consider in building the model. Finally, we can apply our model to completely eradicate Ebola or at least alleviate the current tense situation [4].

\section{How to Restrain the Spread of Ebola}

2.1. Epidemic Model. Considering the production and distribution of drugs and local medical infrastructure, we build a basic epidemic model with maximum treatment capacity. How to restrain the spread of Ebola virus is shown in the following five steps.

Step 1. Build the epidemic model with nonlinear incidence rate and maximum treatment capacity.

Step 2. The total population changes because of the birth rate and natural death rate and is classified into $S(t), E(t), I(t)$, $Q(t)$, and $R(t)$.

Step 3. Build the improved SEIQT epidemic model. 


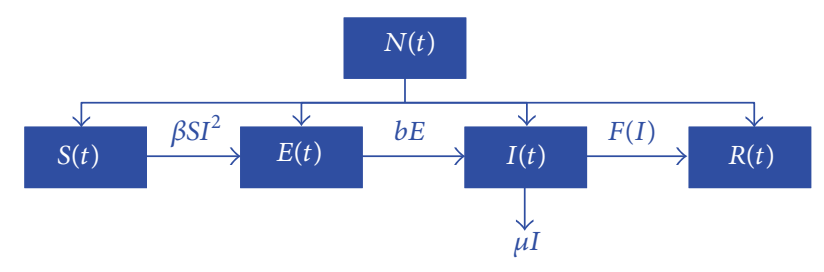

FIgURE 1: Overview of epidemic model.

Step 4. Utilize the equivalent system of equations to figure out the basic reproduction number $R_{0}$. If $R_{0}<1$, the epidemic gets controlled and has a disease-free equilibrium, while it continues to spread and has endemic equilibrium if $R_{0}>1$.

Step 5. Analyze the stability.

2.1.1. Model Preparation. In most of classical epidemic models, the incidence rate is assumed to be $\lambda S I$ ( $\lambda$ is contact rate) which is a bilinear function of $S$ and $I$. However, in fact, many infectious diseases have periodic fluctuations that might be caused by some external factors such as age structure, seasonal variations, and time lag or resulted from nonlinear incidence rate. To fit the actual situation and simplify the model, it is proper to use nonlinear incidence rate. Liu used it in the form of $\beta S^{p} I^{q}(p, q>0)[5,6]$. In this paper, we set $p=1$ and $q=2$.

2.1.2. Model Establishment. We assume that there is no birth rate or natural death rate and the total population is fixed. The process of modeling is shown in Figure 1.

Build simultaneous differential equations:

$$
\begin{aligned}
S^{\prime}(t) & =-\beta S I^{2}, \\
E^{\prime}(t) & =\beta S I^{2}-b E, \\
I^{\prime}(t) & =b E-\mu I-F(I), \\
R^{\prime}(t) & =F(I),
\end{aligned}
$$

associated with maximum treatment capacity:

$$
\begin{aligned}
& S^{\prime}(t)=-\beta S I^{2}, \\
& E^{\prime}(t)=\beta S I^{2}-b E, \\
& I^{\prime}(t)=b E-\mu I-k I, \\
& R^{\prime}(t)=k I,
\end{aligned}
$$

where $0 \leq I \leq I_{0}$,

$$
\begin{aligned}
& S^{\prime}(t)=-\beta S I^{2}, \\
& E^{\prime}(t)=\beta S I^{2}-b E, \\
& I^{\prime}(t)=b E-\mu I-k I_{0}, \\
& R^{\prime}(t)=k I_{0},
\end{aligned}
$$

where $I>I_{0}$.

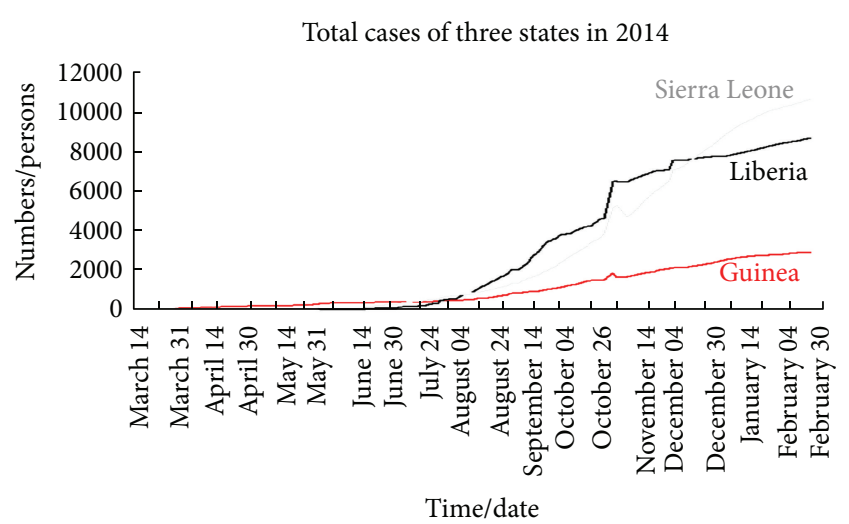

FIgURE 2: Total cases of three states in 2014.

From $S(t)+E(t)+I(t)+R(t)=N(t)$, we can readily get $N^{\prime}(t)=-\mu I$.

At the same time, the function $N(t)$ is the total population of a region or country, the function $S(t)$ is individuals who are susceptible to the disease, the function $E(t)$ is individuals who are infected but without paroxysm, the function $I(t)$ is individuals who are infectious, the function $Q(t)$ is isolators, and the function $R(t)$ is individuals who are removed. The function $F(I)$ is individuals who are cured by the medication, the parameter $\beta$ is proportion of the effective contact in the total population, and the parameter $b$ is proportion of transformation from being infected to being infectious. The parameter $\mu$ is mortality due to illness of the infections, and the parameter $\beta S I^{2}$ is nonlinear incidence rate.

2.2. Improved SEIQR Epidemic Model. First of all, we must introduce the concept of basic reproductive rate. The basic reproduction number (sometimes called basic reproductive ratio and denoted by $R_{0}$ ) of an infection can be thought of as the number of cases that one case generates on average over the course of its infectious period, in an otherwise uninfected population [7].

2.2.1. Data Processing. To analyze the spread rate of Ebola, we collect the data of population and total cases and deaths in Guinea, Liberia, and Sierra Leone from May 27 to November 28 in 2014; see Table 1.

Based on the data in Table 1, we can plot the different graphs which describe the total cases in Sierra Leone, Liberia, and Guinea as shown in Figure 2. Moreover, the graphs in Figures 3-5 reflect the total cases and deaths in those three countries. It is obvious that both numbers have increased rapidly since August.

2.2.2. Model Preparation. To fight against Ebola, infectious individuals tend to be isolated to control the spread of the disease, so as to form a separate group known as isolators. We introduce isolators $Q(t)$ to the model, expand the previous model to SEIQR model, and consider the birth rate $\delta$ and natural death rate $\eta$. Let $\mu_{1}$ and $\mu_{2}$ represent diseased death rate of infectious patients and isolators, respectively, let $b$ 
TABLE 1: The number* of population and total cases and deaths in three countries.

\begin{tabular}{|c|c|c|c|c|c|c|c|c|c|}
\hline \multirow{2}{*}{$\begin{array}{l}\text { Area } \\
\text { Month }\end{array}$} & \multicolumn{3}{|c|}{ Guinea } & \multicolumn{3}{|c|}{ Liberia } & \multicolumn{3}{|c|}{ Sierra Leone } \\
\hline & Population & Total cases & Total deaths & Population & Total cases & Total deaths & Population & Total cases & Total deaths \\
\hline May 27, 2014 & 11.2 million & 281 & 186 & 4.3 million & 12 & 9 & 6.1 million & 16 & 5 \\
\hline June 24, 2014 & 11.2 million & 390 & 270 & 4.3 million & 51 & 34 & 6.1 million & 158 & 34 \\
\hline July 27, 2014 & 11.2 million & 460 & 339 & 4.3 million & 329 & 156 & 6.1 million & 533 & 233 \\
\hline August 26, 2014 & 11.2 million & 648 & 430 & 4.3 million & 1378 & 694 & 6.1 million & 1026 & 422 \\
\hline September 25, 2014 & 11.2 million & 1103 & 668 & 4.3 million & 3564 & 1922 & 6.1 million & 2120 & 561 \\
\hline October 24, 2014 & 11.2 million & 1598 & 981 & 4.3 million & 6253 & 2704 & 6.1 million & 4017 & 1341 \\
\hline November 23, 2014 & 11.2 million & 2134 & 1260 & 4.3 million & 7168 & 3016 & 6.1 million & 6599 & 1398 \\
\hline December 28, 2014 & 11.2 million & 2597 & 1607 & 4.3 million & 7862 & 3384 & 6.1 million & 9004 & 2582 \\
\hline
\end{tabular}

${ }^{*}$ The date in this table is derived from http://www.who.int/mediacentre/news/ebola/23-october-2014/en/.

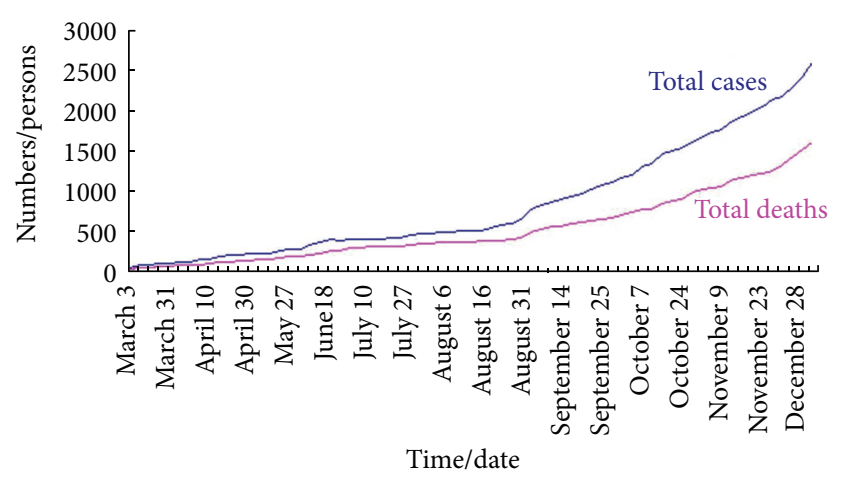

Figure 3: Total cases and deaths in Guinea.

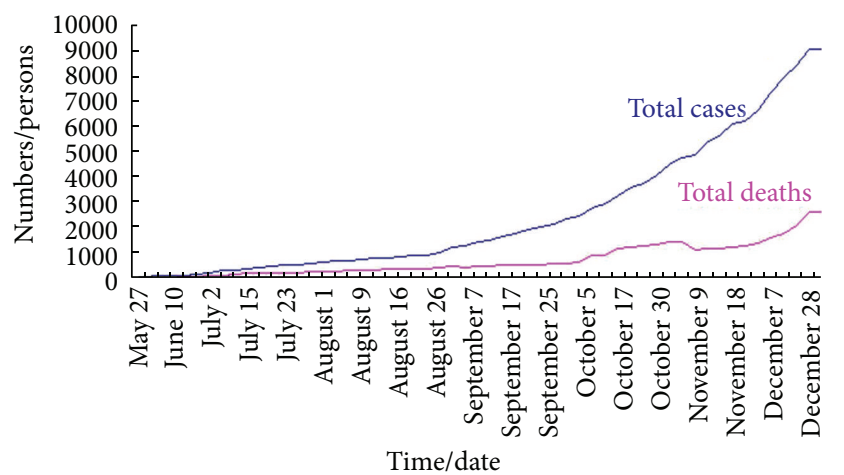

FIGURE 4: Total cases and deaths in Sierra Leone.

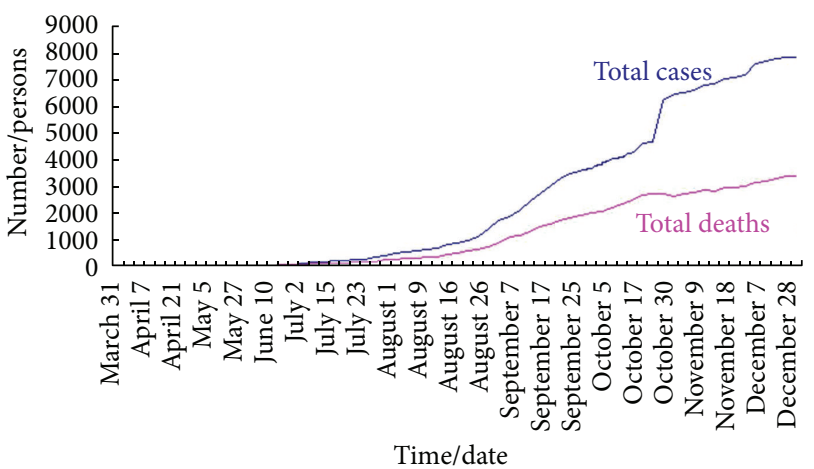

Figure 5: Total cases and deaths in Liberia. represent the proportion of transformation from $E(t)$ to $I(t)$, and let $\varepsilon$ represent the proportion of transformation from $I(t)$ to $Q(t)$. Combined with $\beta S I^{2}$ and maximum treatment capacity, the modifying process is shown in Figure 6.

Also build simultaneous differential equations:

$$
\begin{aligned}
& S^{\prime}(t)=\delta N-\beta S I^{2}-\eta S, \\
& E^{\prime}(t)=\beta S I^{2}-(b+\eta) E, \\
& I^{\prime}(t)=b E-\left(\mu_{1}+\varepsilon+\eta\right) I-F(I), \\
& Q^{\prime}(t)=\varepsilon I-\left(\mu_{2}+\eta\right) Q-F(Q), \\
& R^{\prime}(t)=F(I)+F(Q)-\eta R,
\end{aligned}
$$

where

$$
\begin{gathered}
F(I)= \begin{cases}k I, & 0 \leq I \leq I_{0}, \\
k I_{0}, & I>I_{0},\end{cases} \\
F(Q)= \begin{cases}k Q, & 0 \leq Q \leq I_{0}, \\
k Q_{0}, & Q>Q_{0} .\end{cases}
\end{gathered}
$$

We can find that $S(t)+E(t)+Q(t)+I(t)+R(t)=N(t)$ and $N^{\prime}(t)=(\varepsilon-\eta) N-\mu_{1} I-\mu_{2} Q$.

2.2.3. Model Solution and Analysis. Motivated by the works of $\mathrm{Du}$ and $\mathrm{Xu}[8]$ and Sun and $\mathrm{Ma}$ [9] and the discussions above, we simplify the model by the equivalent system of equations with (4) as follows:

$$
\begin{aligned}
& S^{\prime}(t)=\delta N-\beta S I^{2}-\eta S, \\
& E^{\prime}(t)=\beta S I^{2}-(b+\eta) E, \\
& I^{\prime}(t)=b E-\left(\mu_{1}+\varepsilon+\eta\right) I-F(I) .
\end{aligned}
$$

Then system (6) has a positive invariant set $\Pi=\{(S, E, I) \in$ $\left.R_{+}^{3} \mid 0 \leq S+E+I \leq \delta N / \eta\right\}$.

We obtain the expression of $R_{0}$ :

$$
R_{0}=\frac{b \beta \delta N}{\eta(\eta+b)\left(\eta+\mu_{1}+\varepsilon+k\right)} .
$$




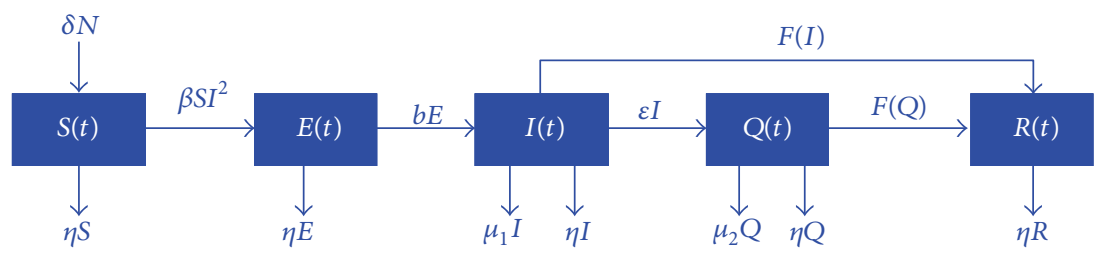

Figure 6: Overview of improved epidemic model.

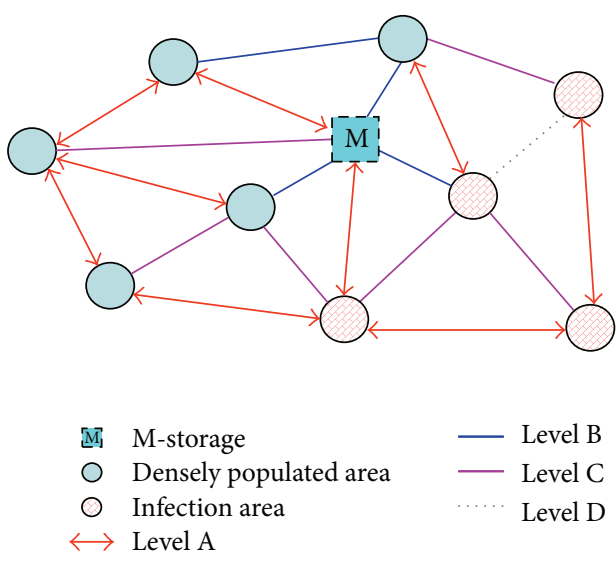

Figure 7: The pictorial diagram of the model.

If $R_{0}<1$, then epidemic gets controlled and system (6) has a disease-free equilibrium $E_{0}$. If $R_{0}>1$, then epidemic continues to spread and system (6) has an endemic equilibrium $E^{*}$.

Therefore, decreasing the basic reproduction number is one of the effective ways to eradicate Ebola or control the development of epidemic [10-12]. We can do it from the following aspects:

(i) Increase the value of $k$ : the speed of drugs production and distribution will affect the number of people being cured. Speeding up the drug production as well as distributing systemically is a powerful control method.

(ii) Decrease the value of $\beta$ : in this condition, the basic reproduction number will be reduced correspondingly. We will reduce the chances that the Ebola virus carriers contact the susceptible person.

(iii) Increase the value of $\varepsilon$ : we can insulate the Ebola virus carriers from other susceptible persons.

\section{How to Distribute Drugs Faster and More Reasonable}

3.1. The Optimal Route Model and M-S Transportation Assignment Model. This model is to establish how to distribute drugs quickly and reasonably. The aim of the first day is acquiring initial data of each infected district, according to the number of the infected people of each district to distribute drugs [13-15] and the number of susceptible people assigned vaccine. On each of the following days, we need to predict the number of changes to the distribution of drugs, according to the model for the infected and susceptible cases [16-19].

The pictorial diagram of the model is given in Figure 7.

(i) The Optimal Route Model. We design the transport speed without resistance as $v$. Here the relationships between the velocity combined with the road level factors and the original ones are

$$
\begin{aligned}
\widetilde{v}_{i} & =\mu_{i} v, \quad \text { where } i=A, B, C, D, E, \\
\mu_{A} & =1, \\
\mu_{B} & =0.8, \\
\mu_{C} & =0.5, \\
\mu_{D} & =0.1, \\
\mu_{E} & =0.000001 .
\end{aligned}
$$

First, we use two matrixes which contain the information of M-S roads' and each of the area roads' level data:

$$
\begin{gathered}
U\left(M-S \longrightarrow m_{i}\right), \\
U^{\prime}\left(m_{i} \longrightarrow m_{j}\right), \\
U=\left(\begin{array}{c}
\mu^{1} \\
\mu^{2} \\
\vdots \\
\mu^{n}
\end{array}\right), U^{\prime}=\left(\begin{array}{cccc}
\mu^{11} & \mu^{12} & \cdots & \mu^{1 n} \\
\mu^{21} & \mu^{22} & \cdots & \mu^{2 n} \\
\vdots & \vdots & & \vdots \\
\mu^{n 1} & \mu^{n 2} & \cdots & \mu^{n n}
\end{array}\right) .
\end{gathered}
$$


The velocity combined with the road level factors can be calculated:

$$
\begin{aligned}
& \widetilde{V}\left(M-S \longrightarrow m_{i}\right), \\
& \widetilde{V}^{\prime}\left(m_{i} \longrightarrow m_{j}\right),
\end{aligned}
$$

$$
\widetilde{V}=U \cdot v=\left(\begin{array}{c}
\mu^{1} v \\
\mu^{2} v \\
\vdots \\
\mu^{n} v
\end{array}\right), \widetilde{V}^{\prime}=U^{\prime} \cdot v=\left(\begin{array}{cccc}
\mu^{11} v & \mu^{12} v & \cdots & \mu^{1 n} v \\
\mu^{21} v & \mu^{22} v & \cdots & \mu^{2 n} v \\
\vdots & \vdots & & \vdots \\
\mu^{n 1} v & \mu^{n 2} v & \cdots & \mu^{n n} v
\end{array}\right) .
$$

We can also get the distance between the M-S and each area:

$$
\begin{gathered}
D\left(M-S \longrightarrow m_{i}\right) \\
L\left(m_{i} \longrightarrow m_{j}\right) \\
D=\left(\begin{array}{c}
d_{1} \\
d_{2} \\
\vdots \\
d_{n}
\end{array}\right), L=\left(\begin{array}{cccc}
l_{11} & l_{12} & \cdots & l_{1 n} \\
l_{21} & l_{22} & \cdots & l_{2 n} \\
\vdots & \vdots & & \vdots \\
l_{n 1} & l_{n 2} & \cdots & l_{n n}
\end{array}\right) .
\end{gathered}
$$

After that, we can obtain shortest time data of each routine. Assume that $A \otimes B$ means two matrix elements' phase in the corresponding phase, where

$$
\begin{aligned}
& T=D \otimes \widetilde{V}=\left(\begin{array}{c}
\frac{d_{1}}{\mu^{1} v} \\
\frac{d_{2}}{\mu^{2} v} \\
\vdots \\
\frac{d_{n}}{\mu^{n} v}
\end{array}\right), \\
& T^{\prime}=L \otimes \widetilde{V}^{\prime}=\left(\begin{array}{cccc}
\frac{l_{11}}{\mu^{11} v} & \frac{l_{12}}{\mu^{12} v} & \cdots & \frac{l_{1 n}}{\mu^{1 n} v} \\
\frac{l_{21}}{\mu^{21} v} & \frac{l_{22}}{\mu^{22} v} & \cdots & \frac{l_{2 n}}{\mu^{2 n} v} \\
\vdots & \vdots & \\
\frac{l_{n 1}}{\mu^{n 1} v} & \frac{l_{n 2}}{\mu^{n 2} v} & \cdots & \frac{l_{n n}}{\mu^{n n} v}
\end{array}\right) .
\end{aligned}
$$

Then we figure out the best routine by using dynamic programming. We design the transport time between area $k$ and area $i$ (destination) as $t_{k i}^{\prime}$, where $k=1,2, \ldots, n, 1 \leq x_{j} \leq$ $n, x_{j} \neq k, x_{j} \neq i$.

When the routine contains one area road,

$$
t_{k i}^{\prime(1)}=T^{\prime}(i, k) \text {. }
$$

When the routine contains two area roads,

$$
t_{k i}^{\prime(2)}=T^{\prime}\left(i, x_{1}\right)+T^{\prime}\left(x_{1}, k\right)
$$

and so on.

When the routine contains zero area roads,

$$
t_{k i}^{\prime(n-1)}=T^{\prime}\left(i, x_{1}\right)+\cdots+T^{\prime}\left(x_{n-1}, k\right) .
$$

Next we get the shortest transport time between arbitrary area and destination:

$$
t_{k}^{\prime}= \begin{cases}0, & k=i, \\ \min \left\{t_{k i}^{\prime(1)}, t_{k i}^{\prime(2)}, \ldots, t_{k i}^{\prime(n-1)}\right\}, & k \neq i .\end{cases}
$$

Finally, we can use Dijkstra Matrix Algorithm to get the shortest time on the routine between every two areas:

$$
\begin{gathered}
D^{(g)}=\left(\begin{array}{cccc}
t_{11}^{\prime} & t_{12}^{\prime} & \cdots & t_{1 n}^{\prime} \\
t_{21}^{\prime} & t_{22}^{\prime} & \cdots & t_{2 n}^{\prime} \\
\vdots & \vdots & & \vdots \\
t_{n 1}^{\prime} & t_{n 2}^{\prime} & \cdots & t_{n n}^{\prime}
\end{array}\right), \\
\text { where } g=\left[\frac{\lg (n-1)}{\lg 2}\right] .
\end{gathered}
$$

The shortest time between M-S and every destination can be figured out: $t_{i}=\min \left(T(k)+t_{k}^{\prime}\right)$. Of course the best routine can also be listed: $H \rightarrow m_{k} \rightarrow \cdots \rightarrow m_{i}$.

The parameter $m_{i}$ denotes the population of each area, $l_{i j}$ denotes the distance between each area, $M$ denotes the drug transport terminal, and $d_{i}$ denotes the distance between terminal and each area.

(ii) M-S Transportation Assignment Model. The virus will infect all the time; every day only deliver a certain amount of drug in order to guarantee the timeliness of delivery. Given that the traffic is limited, we can only dispatch the drug from M-S once a day. Hence the distribution of drugs and vaccines should be allotted according to the infection number and arrival time [20-22]. 


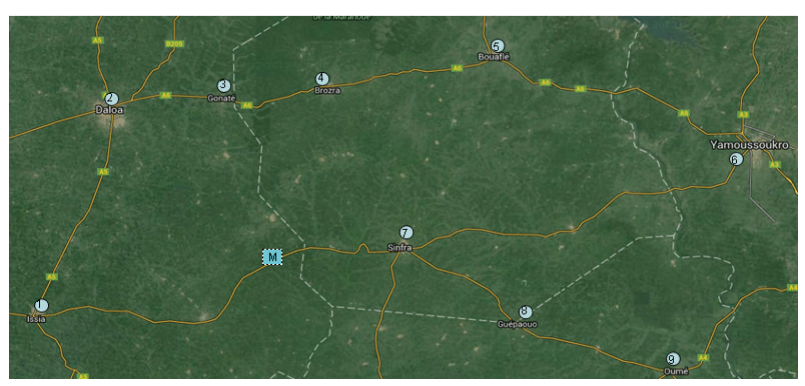

Figure 8: An area of Ivory Coast.

According to the model preparation, we can easily know that

$$
\begin{aligned}
\alpha_{i}(t)= & \frac{I_{i}\left(t+t_{i}\right)}{I\left(t+t_{i}\right)} \cdot \gamma_{1}, \\
\beta_{i}(t)= & \frac{S_{i}\left(t+t_{i}\right)}{S\left(t+t_{i}\right)} \cdot \gamma_{2}, \\
\theta_{1}(t)= & \theta_{1}(0)+\lambda_{1} \eta_{1} \cdot\left(\left[\frac{t}{24}\right]+1\right)-\sigma_{1} \gamma_{1} \\
& \cdot\left(\left[\frac{t}{24}\right]+1\right), \\
\theta_{2}(t)= & \theta_{2}(0)+\lambda_{2} \eta_{1} \cdot\left(\left[\frac{t}{24}\right]+1\right)-\sigma_{2} \gamma_{1} \\
& \cdot\left(\left[\frac{t}{24}\right]+1\right), \\
\frac{\gamma_{1}}{\gamma_{2}}= & \frac{I(t)+Q(t)}{\phi \cdot S(t)} \cdot \varepsilon, \\
\gamma_{1}+\gamma_{2} \leq & \gamma,
\end{aligned}
$$

where $0 \leq \lambda_{1}, \lambda_{2}, \sigma_{1}, \sigma_{2} \leq 1, i=1,2, \ldots, n$.

According to our previously established model, we select the Ivory Coast in an area ( 9 in densely populated areas, a drug storage station) for data simulation. Limited by lack of data search, part of the data (population, prevalence, drug and vaccine production, and storage efficiency) in accordance with the reality of the situation is assumed; see figure position distribution and geographic condition (Figure 8 winning note $\mathrm{M}$ in place of $\mathrm{M}-\mathrm{S}$ ).

According to the map information, we use the transport model to calculate the data. We can get the shortest time by the M-S transport of drugs and vaccines to 9 of this densely populated area with Table 2 . The basic transport rate is $v=$ $60 \mathrm{~km} / \mathrm{h}$.

The most efficient routine is in Table 3.

Based on the data in each region, the initial drug inventory is 5000 units. In this inventory, 2000 units are available every day and 3000 units are used to make vaccines. Besides, 4000 units can be transported when the virus infection coefficient $\varphi=0.8$, and $\varepsilon=1 \times 10^{6}$. Because of insufficient data, the above data and population data and the number of patients are assumed. Pharmaceutical distribution plans of each area can be seen in Tables 4 and 5 .

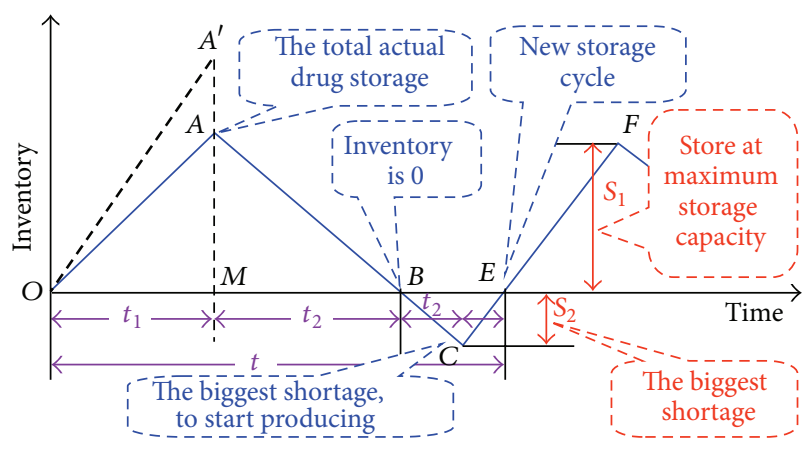

FIGURE 9: Storage capacity of the model with allowed shortages.

From the simulation results, we spend a total of 42 days on complete control of the epidemic. The number of infections in the region is not on the increase. Then the supplies of vaccine and drug need to be sustained.

The parameter $\alpha_{i}(t)$ is the amount of drugs distribution in each region, $\beta_{i}(t)$ is the amount of vaccine distribution in each region, $\gamma$ is the massive daily freight, $\varphi$ is the spread of the virus, $\varepsilon$ is the equilibrium coefficient, $\gamma_{1}$ is the daily traffic volume of drugs, and $\gamma_{2}$ is the daily traffic volume of vaccine.

3.2. Medicine Storage and Transport Model. In this section, we will find what kind of storage solution can make the minimum total cost. Given the drug in the corresponding point of storage, we set up the storage model, then we integrate transport vaccine and drugs production costs, and we can get the minimum total cost solution.

3.2.1. Model Preparation. Combining with the general economic ordering quantity model, we should not only consider the relationship between the transport from the pharmaceutical production department to storage and the affected areas needing drugs but also allow the inventory shortage situation. The rate of transport of drugs is $P$, and the rate of drug demand in the affected area is $D(P>D)$. Production sector starts to deliver drugs to storage starting from 0 . Then at time $t_{1}$, the actual rate of $P$ and $D$ is increasing; after that, the demand reaches the maximum shortage. After the maximum shortage, we restore supply from that point to supplement shortage and start a new cycle for the drug store. Figure 9 shows a schematic view of the corresponding period [23-27].

3.2.2. Model Establishing and Solving. We suppose that a cycle length of time for storage is $t_{1}+t_{2}+t_{3}+t_{4}$ and use OC, CC, and SC to express preparation cost, storage cost, and shortage cost in a storage cycle, respectively. TC represents the average total cost per unit of time.

By analysis, OC, CC, and SC can be given as

$$
\begin{aligned}
& \mathrm{OC}=C_{D}, \\
& \mathrm{CC}=\frac{1}{2} S_{1} C_{P}\left(t_{1}+t_{2}\right), \\
& \mathrm{SC}=\frac{1}{2} S_{2} C_{S}\left(t_{3}+t_{4}\right) .
\end{aligned}
$$


TABLE 2: The shortest time of the transport line.

\begin{tabular}{lccccccccc}
\hline Destination & 1 & 2 & 3 & 4 & 5 & 6 & 7 & 8 & 9 \\
\hline Hours & 1.04375 & 0.985417 & 1.516667 & 3.650417 & 3.750417 & 2.770833 & 1.754167 & 2.34375 & 3.079167 \\
\hline
\end{tabular}

TABLE 3: The most efficient routine.

\begin{tabular}{lccccccc}
\hline Destination & 1 & 2 & 3 & 4 & 5 & 6 & 7 \\
\hline Routine & $M \rightarrow 1$ & $M \rightarrow 2$ & $M \rightarrow 3$ & $M \rightarrow 1 \rightarrow 2 \rightarrow 3$ & $M \rightarrow 1 \rightarrow 2 \rightarrow 3 \rightarrow 4 \rightarrow 5$ & $M \rightarrow 6 M \rightarrow 7$ & $M \rightarrow 7 \rightarrow 8 M \rightarrow 7 \rightarrow 8 \rightarrow 9$ \\
\hline
\end{tabular}

TABLE 4: Transport schedule on the first day.

\begin{tabular}{lcccc}
\hline City & Population & Patients & Drugs & Vaccine \\
\hline 1 & 511154 & 112 & 41 & 471 \\
2 & 12211 & 11 & 5 & 14 \\
3 & 121233 & 0 & 0 & 132 \\
4 & 56454 & 0 & 0 & 55 \\
5 & 64441 & 1 & 1 & 63 \\
6 & 2023756 & 2133 & 767 & 1930 \\
7 & 68782 & 12 & 65 & 161 \\
8 & 124144 & 0 & 0 & 291 \\
9 & 468444 & 0 & 0 & 457 \\
\hline
\end{tabular}

TABle 5: Transport schedule when the epidemic is controlled.

\begin{tabular}{lcccc}
\hline City & Population & Patients & Drugs & Vaccine \\
\hline 1 & 511154 & 131 & 48 & 495 \\
2 & 12211 & 11 & 5 & 14 \\
3 & 121233 & 0 & 0 & 139 \\
4 & 56454 & 0 & 0 & 68 \\
5 & 64441 & 0 & 0 & 79 \\
6 & 2023756 & 1683 & 662 & 2513 \\
7 & 68782 & 4 & 663 & 348 \\
8 & 124144 & 0 & 0 & 700 \\
9 & 468444 & 0 & 0 & 3337 \\
\hline
\end{tabular}

So the average total cost per unit of time can be obtained as

$$
\begin{aligned}
\mathrm{TC} & =\frac{\mathrm{OC}+\mathrm{CC}+\mathrm{SC}}{t_{1}+t_{2}+t_{3}+t_{4}} \\
& =\frac{C_{D}+0.5 \cdot S_{1} C_{P}\left(t_{1}+t_{2}\right)+0.5 \cdot S_{2} C_{S}\left(t_{3}+t_{4}\right)}{t_{1}+t_{2}+t_{3}+t_{4}} .
\end{aligned}
$$

From Figure 9, we can get

$$
\begin{aligned}
S_{1} & =(P-D) t_{1}=D t_{2} \longrightarrow \\
t_{1} & =\frac{D}{P-D} t_{2}, \\
t_{1}+t_{2} & =\frac{P}{P-D} t_{2},
\end{aligned}
$$

$$
\begin{aligned}
S_{2} & =(P-D) t_{4}=D t_{3} \longrightarrow \\
t_{4} & =\frac{D}{P-D} t_{3}, \\
t_{3}+t_{4} & =\frac{P}{P-D} t_{3}, \\
t_{1}+t_{2}+t_{3}+t_{4} & =\frac{P}{P-D}\left(t_{2}+t_{3}\right), \\
Q & =D\left(t_{1}+t_{2}+t_{3}+t_{4}\right) \\
& =\frac{P D}{P-D}\left(t_{2}+t_{3}\right) .
\end{aligned}
$$

Restoring data, each length of time can be given as

$$
\begin{aligned}
t_{3}^{*} & =\sqrt{\frac{2 C_{D} C_{S}(1-D / P)}{D C_{S}\left(C_{P}+C_{S}\right)}}, \\
t_{2}^{*} & =\sqrt{\frac{2 C_{D} C_{S}(1-D / P)}{D C_{P}\left(C_{P}+C_{S}\right)}}, \\
Q^{*} & =\sqrt{\frac{2 C_{D}\left(C_{P}+C_{S}\right) D}{C_{P} C_{S}(1-D / P)}}, \\
S_{1}^{*} & =D t_{2}^{*}=\sqrt{\frac{2 D C_{D} C_{S}(1-D / P)}{C_{P}\left(C_{P}+C_{S}\right)}}, \\
S_{2}^{*} & =D t_{3}^{*}=\sqrt{\frac{2 D C_{D} C_{S}(1-D / P)}{C_{S}\left(C_{P}+C_{S}\right)} .} .
\end{aligned}
$$

With generation into the TC we can get the minimum cost:

$$
\mathrm{TC}^{*}=\sqrt{\frac{2 D C_{D} C_{S} C_{P}(1-D / P)}{C_{P}+C_{S}}}
$$

where $C_{D}$ denotes the cost of preparations before transporting drugs, $C_{P}$ means storage fee of unit drugs in unit time, and $C_{S}$ means economic losses caused by drug shortages in unit time.

After obtaining the sum cost of the average fee, storage fee, and loss fee in the unit of time, the final total cost can be obtained after adding the corresponding medicine manufacturing cost and transportation cost. 


\section{Conclusions}

In summary, we can enlarge the drugs and vaccine supply to control the epidemic. As we can see, the index of $\gamma$ is over one, which means increasing $A$ is more efficient in decreasing the virus spread velocity [28]. In addition, when the epidemic was controlled, focusing on the medicine research can be better than the expansion of production on drugs and vaccine. If we can provide more helpful things to people, we do believe that Ebola will be erased in this world [29-33]. By the way, reducing the cost in producing as well as putting more money on the researching is a sustainable plan to face the future that the viruses will become variants.

\section{Competing Interests}

The authors declare that there is no conflict of interests regarding the publication of this paper.

\section{Acknowledgments}

The work was supported in part by the Natural Science Foundation of Anhui Province of China under Grant no. KJ2013B105, the Natural Science Foundation for the Higher Education Institutions of Anhui Province of China under Grant no. KJ2015A331, the National Natural Science Foundation of China, no. 11301001, the Excellent Youth Scholars Foundation, and the Natural Science Foundation of Anhui Province of China under Grant no. 2013SQRL030ZD.

\section{References}

[1] Media Centre, "Summary report of a WHO High-level meeting on Ebola vaccines access and financing," Tech. Rep., 2014, http://www.who.int/mediacentre/news/ebola/23-october-2014/ en/.

[2] The concept of basic reproductive rate, http://en.wikipedia.org/ wiki/Basic_reproduction_number.

[3] WHO, "Cholera: global surveillance summary, 2008," Weekly Epidemiological Record, vol. 84, no. 31, pp. 309-324, 2009.

[4] G. H. Li and J. Bai, "The study on an epidemic model with nonlinear incidence and piecewise treatment function," Mathematics in Practice and Theory, vol. 44, no. 21, pp. 228-236, 2014.

[5] W. M. Liu, S. A. Levin, and Y. Iwasa, "Influence of nonlinear incidence rates upon the behavior of SIRS epidemiological models," Journal of Mathematical Biology, vol. 23, no. 2, pp. 187204, 1986.

[6] W. M. Liu, H. W. Hethcote, and S. A. Levin, "Dynamical behavior of epidemiological models with nonlinear incidence rates," Journal of Mathematical Biology, vol. 25, no. 4, pp. 359380, 1987.

[7] A. Pruss-Ustun, R. Bos, F. Gore, and J. Bartram, Safer Water, Better Health: Costs, Benefits and Sustainability of Interventions to Protect and Promote Health, World Health Organization, Geneva, Switzerland, 2008.

[8] Y. K. Du and R. Xu, "Pattern formation in two classes of SIR epidemic models with spatial diffusion," Chinese Journal of Engineering Mathematics, vol. 31, no. 3, pp. 454-462, 2014.
[9] X.-K. Sun and C.-Ch. Ma, "Stability of a class of epidemic model with latent period," Journal of Biomathematics, vol. 29, no. 4, pp. 663-667, 2014.

[10] C. P. Gerba, J. B. Rose, C. N. Haas, and K. D. Crabtree, "Waterborne rotavirus: a risk assessment," Water Research, vol. 30, no. 12, pp. 2929-2940, 1996.

[11] M. M. Marshall, D. Naumovitz, Y. Ortega, and C. R. Sterling, "Waterborne protozoan pathogens," Clinical Microbiology Reviews, vol. 10, no. 1, pp. 67-85, 1997.

[12] T. E. Ford, "Microbiological safety of drinking water: United States and global perspectives," Environmental Health Perspectives, vol. 107, no. 1, pp. 191-206, 1999.

[13] N. J. Ashbolt, "Microbial contamination of drinking water and disease outcomes in developing regions," Toxicology, vol. 198, no. 1-3, pp. 229-238, 2004.

[14] C. Kourenti, P. Karanis, and H. Smith, "Waterborne transmission of protozoan parasites: a worldwide review of outbreaks and lessons learnt," Journal of Water and Health, vol. 5, no. 1, pp. $1-38,2007$.

[15] K. T. Goh, S. H. Teo, S. Lam, and M. K. Ling, "Person-to-person transmission of cholera in a psychiatric hospital," Journal of Infection, vol. 20, no. 3, pp. 193-200, 1990.

[16] M. D. Andersen and N. F. Neumann, "Giardia intestinalis: new insights on an old pathogen," Reviews in Medical Microbiology, vol. 18, no. 2, pp. 35-42, 2007.

[17] J. N. S. Eisenberg, B. L. Lewis, T. C. Porco, A. H. Hubbard, and J. M. Colford Jr., "Bias due to secondary transmission in estimation of attributable risk from intervention trials," Epidemiology, vol. 14, no. 4, pp. 442-450, 2003.

[18] A. A. King, E. L. Ionides, M. Pascual, and M. J. Bouma, "Inapparent infections and cholera dynamics," Nature, vol. 454, no. 7206, pp. 877-880, 2008.

[19] C. T. Codeço, "Endemic and epidemic dynamics of cholera: the role of the aquatic reservoir," BMC Infectious Diseases, vol. 1, article 1, 2001.

[20] S. M. Faruque, I. M. Johirul, A. Q. Shafi et al., "Self-limiting nature of seasonal cholera epidemics: role of host-mediated amplification of phage," Proceedings of the National Academy of Sciences of the United States of America, vol. 21, no. 5-6, pp. 10091060, 1999.

[21] D. M. Hartley, J. G. Morris Jr., and D. L. Smith, "Hyperinfectivity: a critical element in the ability of $V$. cholerae to cause epidemics?" PLoS Medicine, vol. 3, no. 1, pp. 63-69, 2006.

[22] K. Koelle, M. Pascual, and M. Yunus, "Pathogen adaptation to seasonal forcing and climate change," Proceedings of the Royal Society B: Biological Sciences, vol. 272, no. 1566, pp. 971-977, 2005.

[23] K. Koelle, M. Pascual, and M. Yunus, "Serotype cycles in cholera dynamics," Proceedings of the Royal Society B: Biological Sciences, vol. 273, no. 1603, pp. 2879-2886, 2006.

[24] J. H. Tien and D. J. Earn, "Multiple transmission pathways and disease dynamics in a waterborne pathogen model," Bulletin of Mathematical Biology, vol. 72, no. 6, pp. 1506-1533, 2010.

[25] J. M. Hyman, J. Li, and E. Ann Stanley, "The differential infectivity and staged progression models for the transmission of HIV," Mathematical Biosciences, vol. 155, no. 2, pp. 77-109, 1999.

[26] B. G. Wagner and D. J. D. Earn, "Population dynamics of liveattenuated virus vaccines," Theoretical Population Biology, vol. 77, no. 2, pp. 79-94, 2010. 
[27] A. Lajmanovich and J. A. Yorke, "A deterministic model for gonorrhea in a nonhomogeneous population," Mathematical Biosciences, vol. 28, no. 3-4, pp. 221-236, 1976.

[28] X. D. Lin and J. W.-H. So, "Global stability of the endemic equilibrium and uniform persistence in epidemic models with subpopulations," The Journal of the Australian Mathematical Society, Series B: Applied Mathematics, vol. 34, no. 3, pp. 282$295,1993$.

[29] H. B. Guo and M. Y. Li, "Global dynamics of a staged progression model for infectious diseases," Mathematical Biosciences, vol. 28, no. 3-4, pp. 221-236, 1976.

[30] H. Guo, M. Y. Li, and Z. Shuai, "A graph-theoretic approach to the method of global Lyapunov functions," Proceedings of the American Mathematical Society, vol. 136, no. 8, pp. 2793-2802, 2008.

[31] M. Y. Li and Z. Shuai, "Global-stability problem for coupled systems of differential equations on networks," Journal of Differential Equations, vol. 248, no. 1, pp. 1-20, 2010.

[32] A. Berman and R. J. Plemmons, Nonnegative Matrices in the Mathematical Sciences, Academic Press, New York, NY, USA, 1979.

[33] J. K. Hale, Ordinary Differential Equations, Wiley-Interscience, New York, NY, USA, 1969. 


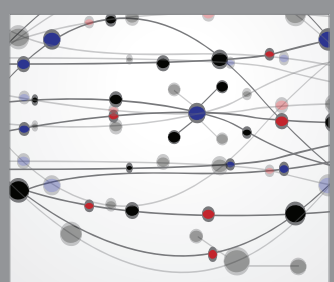

The Scientific World Journal
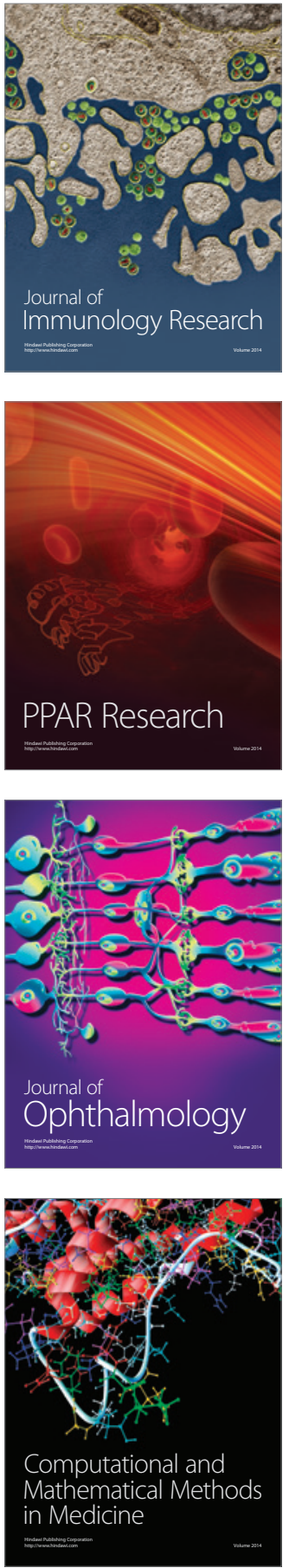

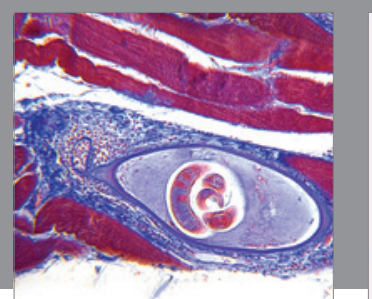

Gastroenterology Research and Practice

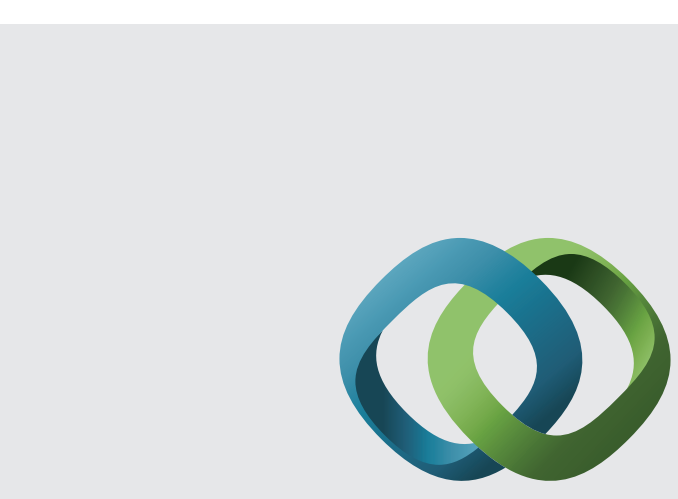

\section{Hindawi}

Submit your manuscripts at

http://www.hindawi.com
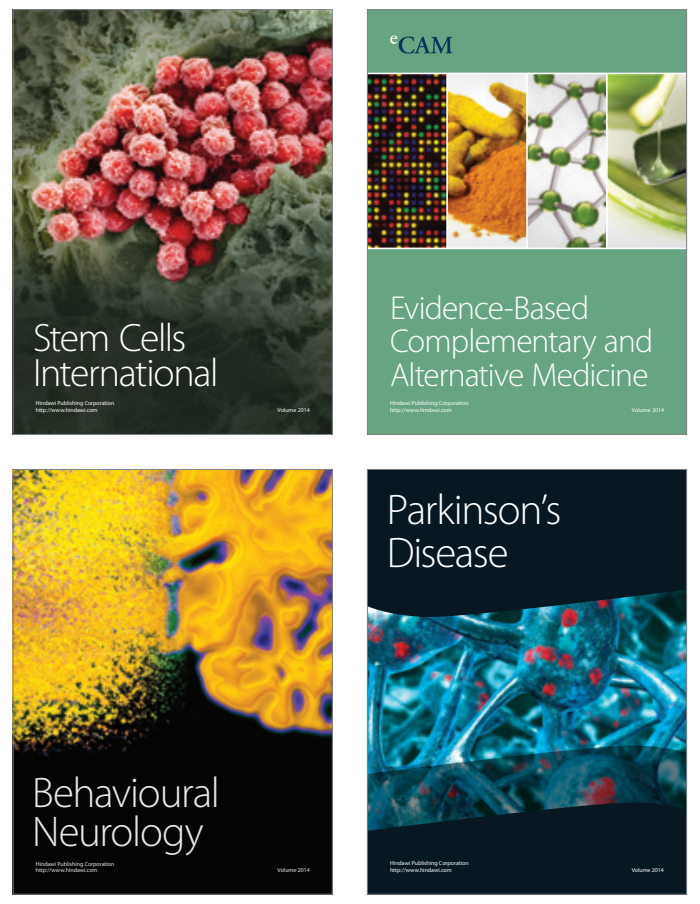
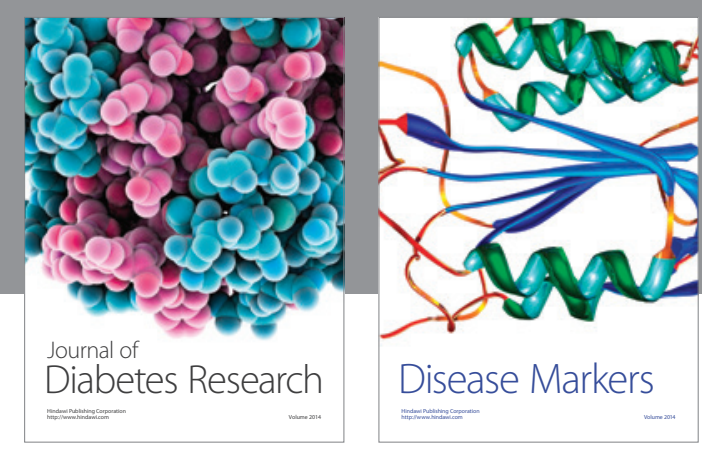

Disease Markers
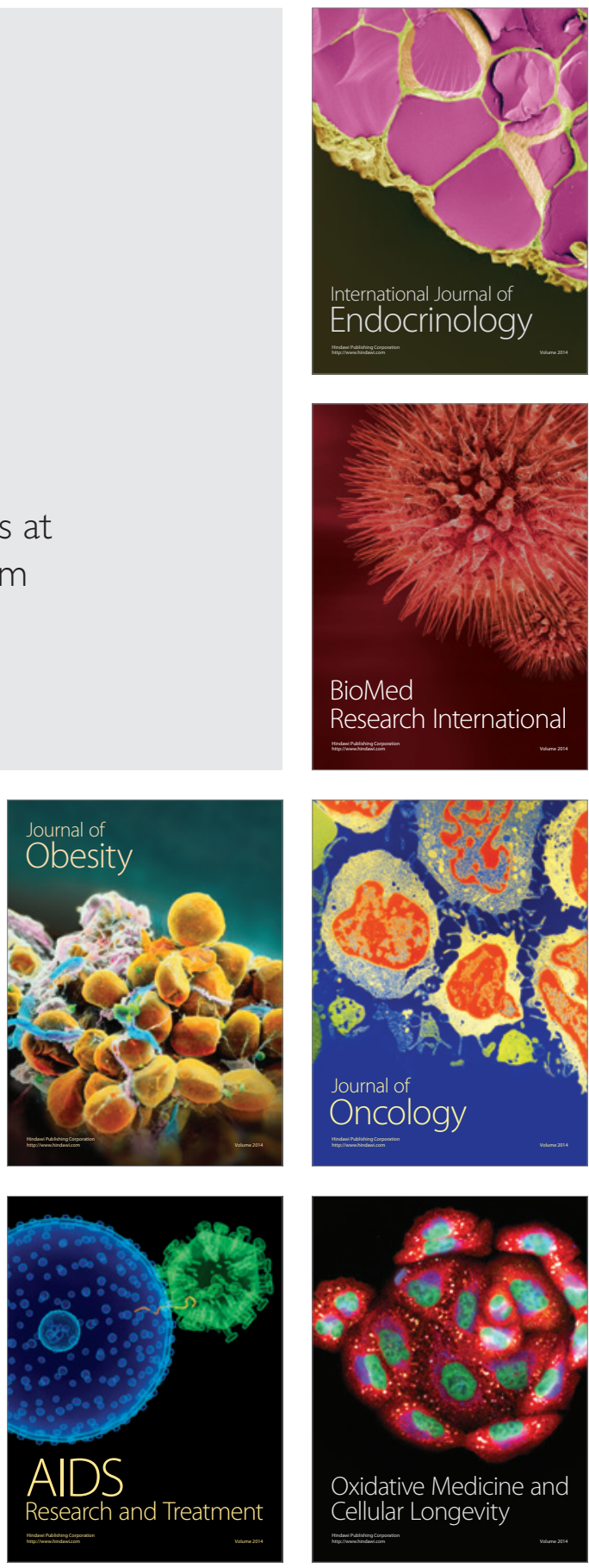